\title{
The effects of body weight-supported treadmill training on static and dynamic balance in stroke patients: A pilot, single-blind, randomized trial
}

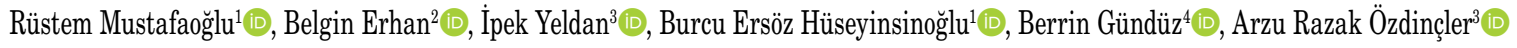 \\ ${ }^{1}$ Department of Neurological Physiotherapy and Rehabilitation, Division of Physiotherapy and Rehabilitation, Istanbul University, Faculty of Health Sciences, Istanbul, Turkey \\ ${ }^{2}$ Department of Physiotherapy and Rehabilitation, Division of Physiotherapy and Rehabilitation, Istanbul Aydın University, Faculty of Health Sciences, Istanbul, Turkey \\ ${ }^{3}$ Department of Physiotherapy and Rehabilitation, Division of Physiotherapy and Rehabilitation, Istanbul University, Faculty of Health Sciences, Istanbul, Turkey \\ ${ }^{4}$ Department of Physical Medicine and Rehabilitation, Istanbul Physical Therapy Rehabilitation Training and Research Hospital, Istanbul, Turkey
}

Received: January 22, 2018 Accepted: June 27, 2018 Published online: August 15, 2018

\begin{abstract}
Objectives: This study aims to compare effectiveness of isolated body weight-supported treadmill training (BWSTT) with conventional and combined training on balance, mobility, and fear of falling in stroke patients.

Patients and methods: Between November 2014 and November 2015, a total of 45 post-stroke patients (32 males, 13 females; mean age $53.1 \pm 13.2$ years; range, 19 to 73 years) were randomly assigned to combined training group (CombTG), conventional training group (CTG), and BWSTT group (BWSTTG). The CombTG received 45 min conventional therapy for five days a week along with 45 min of BWSTT twice a week. The CTG received only conventional therapy for five days a week. The BWSTTG received only BWSTT twice a week. Training duration was six weeks for all groups. Primary outcome measures were the Berg Balance Scale (BBS), affected and non-affected side Single Leg Stance Test (e-SLST/n-SLST), and Timed Up and Go Test (TUG) results. Secondary outcomes were the Falls Efficacy Scale-International (FES-I), Rivermead Mobility Index (RMI), Comfortable 10-m Walk Test (CWT), and Stair Climbing Test (SCT) results.

Results: The mean change of outcome measures demonstrated that the improvements between groups were significantly different among the three groups, except for the CWT ( $\mathrm{p}=0.135)$. In subgroup analysis, except for the RMI and CWT, all primary and secondary outcome measures improved significantly in favor of the CombTG, compared to the CTG and BWSTTG ( $<<0.016)$. However, no statistically significant difference was found in the mean change of the CTG and BWSTTG $(\mathrm{p}>0.05)$.

Conclusion: This study demonstrates that combined training has considerable effects on balance, mobility, and fear of falling parameters, while lower frequency of isolated BWSTT is as much effective as higher frequency of conventional training in ambulatory post-stroke patients.

Keywords: Accidental falls; mobility limitation; postural balance; rehabilitation; stroke.
\end{abstract}

Stroke is one of the most common causes of acquired adult disability. The majority of stroke survivors have mobility difficulties such as poor standing, decreased walking speed, balance disturbances, and increased risk of falls. Improving mobility, walking, and balance are the main goals of stroke rehabilitation. ${ }^{[1]}$ Conventional therapy acts as the critical role and has been shown to be effective for recovery of mobility and gait in post-stroke patients. ${ }^{[2]}$ Body weight-supported treadmill training (BWSTT) has been becoming a more promising intervention for locomotor training in post-stroke rehabilitation. The goal of locomotor training is to perform specific tasks which provoke neuroplasticity, thereby, improving motor control and functional independency. ${ }^{[3]}$ As BWSTT offers more repetitive practice than conventional therapy, it seems to have more advantages to promote motor recovery. ${ }^{[4-6]}$

Static or dynamic balance deficits play critical roles on gait performance among stroke survivors. Although BWSTT has been shown to lead to higher improvement in balance function compared to conventional training (CT) in stroke patients, ${ }^{[7-11]}$ some authors have suggested that BWSTT is not superior to $\mathrm{CT} \cdot{ }^{[4,12,13]}$ Of note, in these studies, the frequency

Corresponding author: İpek Yeldan, PhD, PT. İstanbul Üniversitesi, Sağlık Bilimleri Fakültesi, Fizyoterapi ve Rehabilitasyon Bölümü, 34740 Bakırköy, İstanbul, Turkey. 
of BWSTT and CT were not similar. Although it is known that dosing parameters such as amounts, frequencies, and durations in neurorehabilitation are important issues to prescribe motor therapy, ${ }^{[14,15]}$ there is no consensus on the most beneficial method and the optimal training dose to improve balance function in stroke patients. On the other hand, it is well-known that balance impairment have a profound impact on fear of falling. It has been reported that chronic stroke patients with a history of multiple falls are more likely to experience fear of falling. To the best of our knowledge, although there are some studies investigating the impact of BWSTT on balance confidence, ${ }^{[10,16]}$ there is no study evaluating the level of fear of falling after BWSTT in stroke patients in the literature.

In the present study, we hypothesized that applying combined training would cause better results than CT or BWSTT alone. In addition, the participants who receive $\mathrm{CT}$ with a higher frequency would demonstrate improved performance in the outcome measures, compared to the lower frequency of BWSTT. Therefore, we aimed to compare the effects of CT, BWSTT, and the combination of these interventions (combined training) in improving balance, mobility, and fear of falling in post-stroke patients.

\section{PATIENTS AND METHODS}

This pilot, single-blind, prospective, randomized study included a total of 45 post-stroke patients (32 males, 13 females; mean age $53.1 \pm 13.2$ years; range, 19 to 73 years) between November 2014 and November 2015. The patients were randomly assigned to combined training group (CombTG), conventional training group (CTG), and BWSTT group (BWSTTG). An informed consent was obtained from each patient. The study protocol was approved by the Institutional Review Board (2014/A-23). The study was conducted in accordance with the principles of the Declaration of Helsinki. The Clinical Registration Number is ClinicalTrials.gov ID:NCT02735148. All participants were recruited from a public rehabilitation hospital. The inclusion criteria were as follows: (i) stroke onset at least three months before the study, (ii) age range of 18-75 years old, (iii) to be able to walk $10 \mathrm{~m}$ independently or under supervision, and (iv) to be able to understand all instructions during treatment sessions (Mini-Mental State Examination Score $\geq 24$ points). ${ }^{[17]}$ Exclusion criteria were as follows: (i) a previous stroke, (ii) musculoskeletal disorders causing contracture or limited range of motion in their lower extremities affecting walking, and (iii) severe heart disease or medically uncontrolled hypertension.

A total of 107 patients were assessed for eligibility by two physical medicine and rehabilitation specialists (Figure 1). Of these, 45 patients were found to be eligible for the study. Randomization was performed by random number generator of the Microsoft Office Excel Software, which gives a random number between 0 and 1 to each treatment columns (Group distribution: $0-<0.33=$ CombTG $, 0.33-<0.66=\mathrm{CT}, 0.66-1=$ BWSTT). Sorting of random number row was performed from the largest to the smallest numbers by the sort and filter menu. To ensure a distribution balance among three groups, stratification of treatment assignments was accomplished by the ambulation levels according to the Functional Ambulation Scale (FAS). After randomization, primary and secondary outcome assessments at baseline and after training were performed by a blinded assessor.

\section{Training protocol}

\section{Conventional training}

After the assessment, a rehabilitation program was planned according to each participant's functional level and requirements. The CT program focused on trunk stabilization, weight transfer to the paretic leg, and walking between parallel bars or on the ground. Treatment activities were designed to improve balance, while encouraging the participant to use his or her more paretic lower limb. Verbal and tactile cues were used to encourage symmetrical weight distribution. The training was also complicated by adding arm activities and reaching activities while walking forward, backward and side to side. The CTG received a session lasted $45 \mathrm{~min}$ according to the government's health insurance rules for five week days over six weeks.

\section{Body Weight-Supported Treadmill Training}

The Lokomat system (Hocoma AG, Volketswil, Switzerland) with an integrated treadmill and a motordriven body weight support system with real-time feedback control for precise body weight unloading was used for the BWSTT. For each participant, the body weight portion was ensured by a security belt while walking. Approximately 30 to $40 \%$ of an individual's body weight was supported during the first session and decreased by relative $10 \%$ increments per session as tolerated without substantial knee buckling or toe drag. ${ }^{[18]}$ The velocity of treadmill was adjusted from 1.2-2.6 $\mathrm{km} / \mathrm{h}$ and was set to the maximum speed tolerated by 


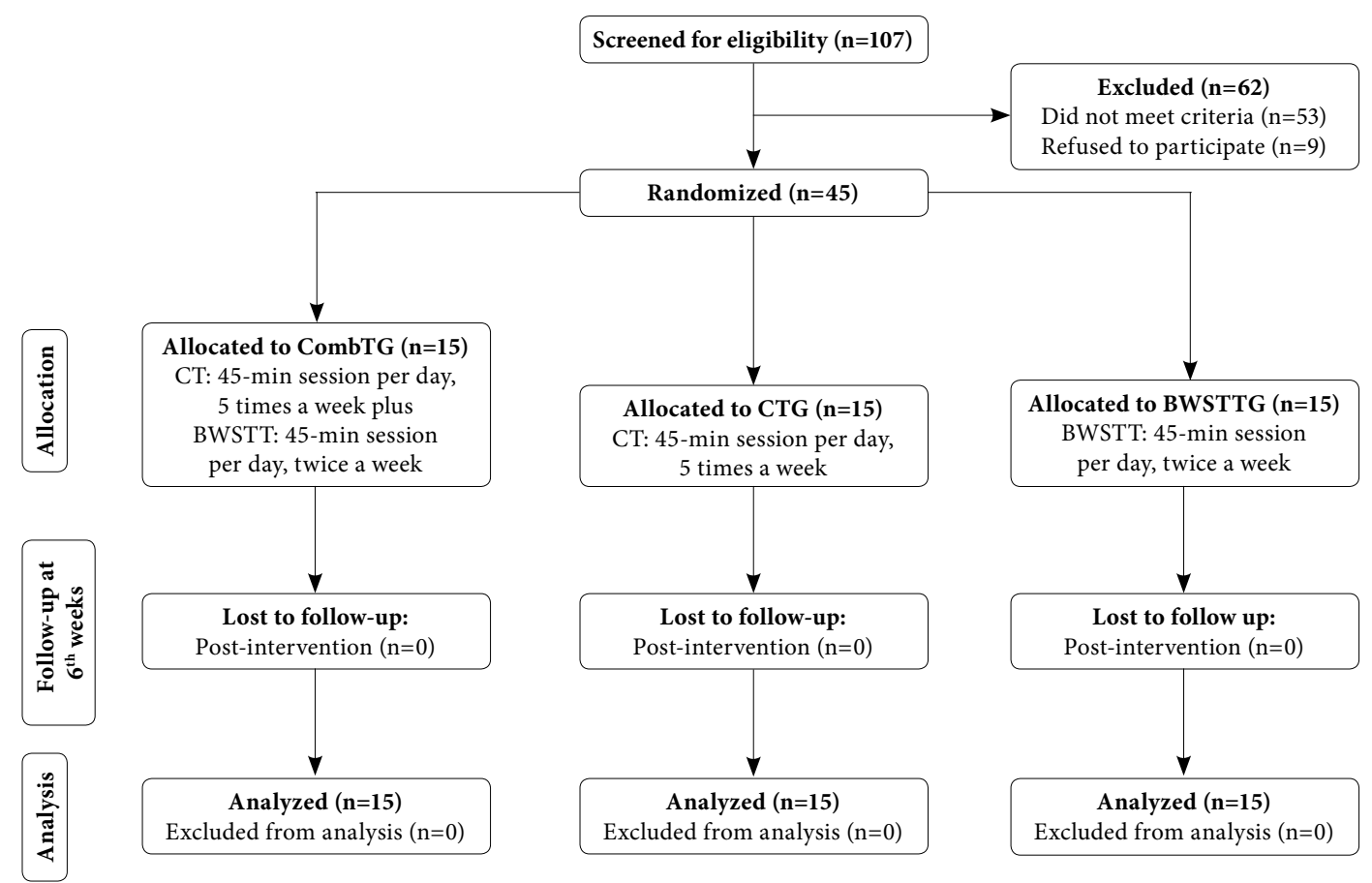

Figure 1. CONSORT flowchart of the study.

CombTG: Combined training group; BWSTT: Body weight supported treadmill training; CTG: Conventional training group; CT: Conventional training; BWSTTG: Body weight supported treadmill training group.

the patient during sessions. ${ }^{[19]}$ Each session included setup, commands, and resting time. Verbal instructions were used for encouragement, but no manual assistance was provided to improve gait pattern. All parameters were individually adjusted for each session. The actual training time was 45 min per session, excluding time required for putting on equipment and operation of the computer. The BWSTTG received 45 min, two nonconsecutive days per week for six-week gait training on the Lokomat. According to the governmental health insurance coverage, the patients received BWSTT twice a week. All patients were invited to the hospital's CT program at the end of study.

\section{Combined training}

Combined training patients received inpatient rehabilitation. The CombTG received BWSTT (Lokomat; $45 \mathrm{~min}$, two non-consecutive days per week for six weeks) combined with CT (45 min, five days per week for six weeks).

\section{Outcome measures}

The outcome measurements were obtained before and immediately after training program. All participants were allowed to use assistive devices during testing.
Demographic and clinical features were recorded for each participant at baseline.

\section{Primary outcomes}

\section{The Berg Balance Scale (BBS)}

This 14-item objective measure was used to assess postural control and balance of the participants. Itemlevel scores of BBS range from 0-4. Total scores of items were used in this study. A higher score indicates a better mobility performance. ${ }^{[20,21]}$

\section{The Single Leg Stance Test (SLST)}

Static balance was measured by SLST. Shortened time to stand on one leg was accepted as a marker for decreased balance function. ${ }^{[22]}$

\section{The Timed Up and Go Test (TUG)}

The TUG was used to assess balance and functional mobility of post-stroke patients. A lower duration indicates better mobility performance. ${ }^{[13,23,24]}$

\section{Secondary outcomes}

\section{The Falls Efficacy Scale-International (FES-I)}

The FES-I was used to assess the fear of falling level of participants about falling during indoor or outdoor activities. It has 16 items scored on a four-point 


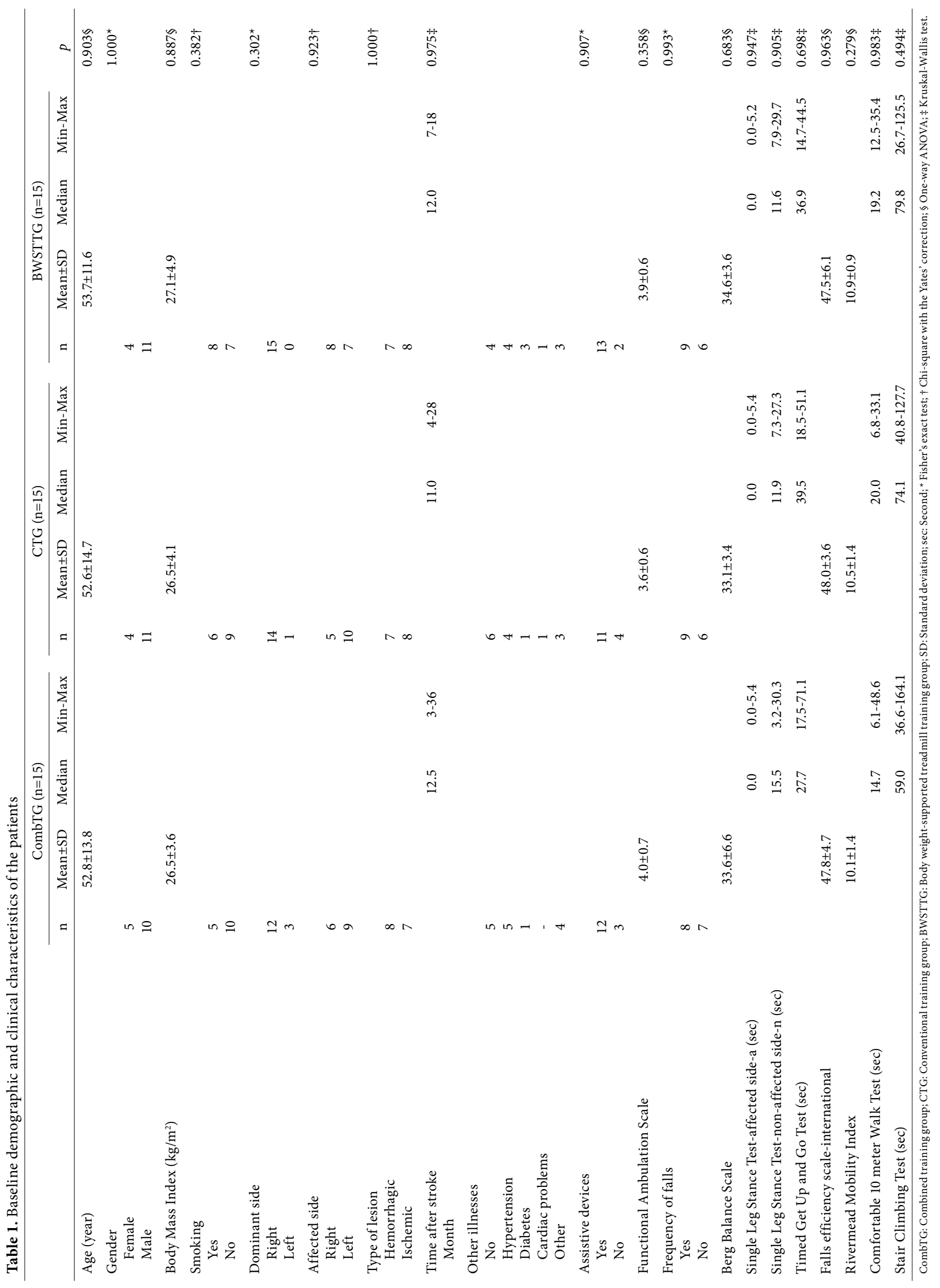


Likert scale. The Turkish validated version of the FES-I was used in this study. A higher score indicates better mobility performance. ${ }^{[25]}$

\section{The Rivermead Mobility Index (RMI)}

The RMI was used to assess the functional mobility of the patients. This 15 -item test is a hierarchical mobility scale including rolling in bed to running. A higher score indicates better mobility performance. ${ }^{[26]}$

\section{The Comfortable 10-m Walk Test (CWT)}

The CWT was used to determine the speed of comfortable walking. The test was applied in a $10-\mathrm{m}$ corridor. At the first meter, a stopwatch was started and stopped, when the patient reached the $10^{\text {th }} \mathrm{m} \cdot{ }^{[27]}$

\section{The Stair Climbing Test (SCT)}

The duration of ascending and descending 10 steps (17 cm of height) was measured in sec with a stopwatch. The patients are allowed to use handrails or assistive devices, if necessary. ${ }^{[23]}$

After three trials for the SLST, CWT, and SCT, the mean of the three trials was recorded.

\section{Statistical analysis}

Statistical analysis was performed using the IBM SPSS version 20.0 software (IBM Corp., Armonk, NY, USA). The normality of data distribution was tested using the Shapiro-Wilk test of normality. Descriptive statistics were expressed in mean and standard deviation (SD), or frequency (\%). Demographic comparisons of the groups were conducted using the one-way analysis of variance (ANOVA) for normally distributed continuous variables and the KruskalWallis test for not normally distributed continuous variables and, also, the chi-square test for categorical variables. As the Shapiro-Wilk tests showed that the SLST-a/n, TUG, CWT, and SCT data were not normally distributed, non-parametric tests (Wilcoxon signedrank test, Kruskal-Wallis test) were applied. The BBS, FES-I, and RMI data met the assumption of normality based on the Shapiro-Wilk test and were analyzed using parametric tests (paired sample t-test, one-way ANOVA). Post-hoc pairwise comparisons were carried out using the Bonferroni test at a significance level of $<0.016$. For the other analyses, a $p$ value of $<0.05$ was considered statistically significant. The effect size (d) was calculated by taking the difference between the means before treatment and after treatment and dividing it by the SD of the same measure before treatment. An effect size of 0.2 was considered small, that of 0.5 was considered moderate, and that of 0.8 was considered large. ${ }^{[28]}$

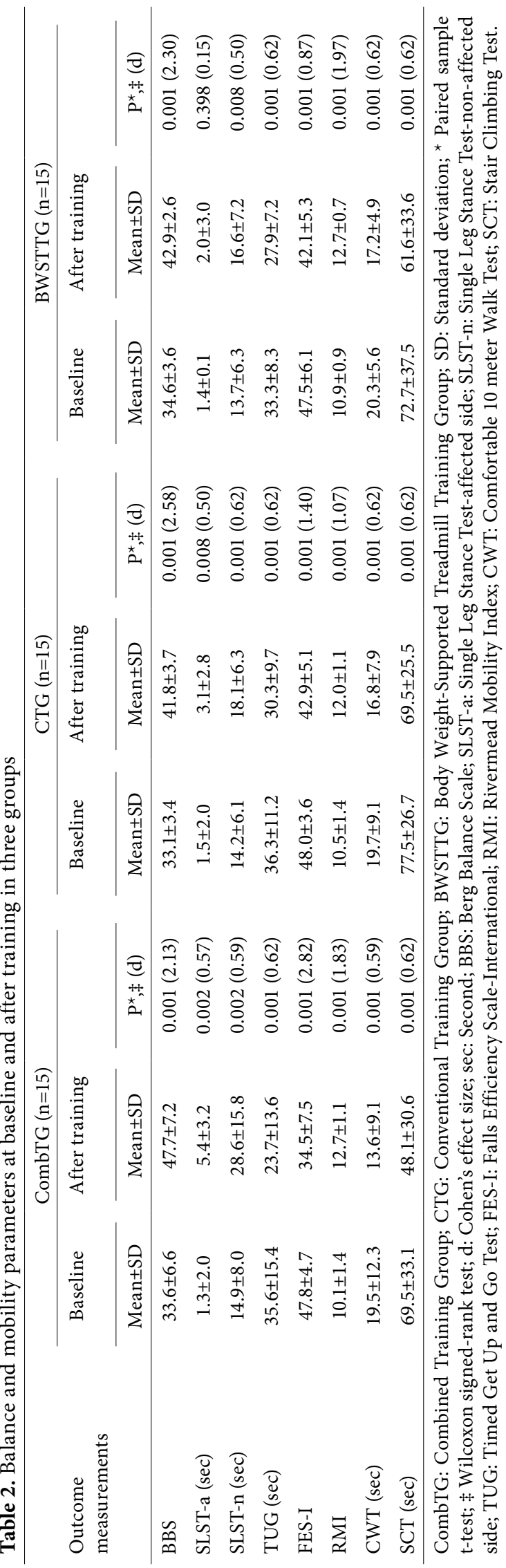




\section{RESULTS}

A total of 107 patients were assessed for eligibility, and 53 of them failed to meet the inclusion criteria, while nine patients refused to participate in the study. No dropout was observed in groups during six-week study period; all 45 patients completed the study (Figure 1). Demographic and clinical features of the study participants are shown in Table 1 . There were no statistically significant differences in demographic and clinical features at baseline (Table 1).

In terms of pre- and post-training, there were statistically significant improvements in the BBS, SLST-affected side (SLST-a), SLST-non-affected side (SLST-n), TUG in the all groups $(\mathrm{p}<0.05)$, except for SLST-a in the BWSTTG after six-week training $(p=0.398)$. The effect size were large in all groups for
BBS and moderate in all groups for SLST-a, SLST-n and TUG. Significant improvements were also determined for the FES-I, RMI, CWT, and SCT in three groups after treatment $(\mathrm{p}=0.001)$. The effect size was large in all groups for the FES-I, RMI, and moderate in CWT and SCT (Table 2).

The mean change in the BBS, SLST-a, SLST-n, TUG, FES-I, RMI, CWT, and SCT scores at baseline versus post-training are represented in Table 3 . The mean changes in primary outcome measures were statistically significant $(\mathrm{p}<0.05)$ among the groups. In subgroup analysis, all primary outcome measures improved significantly in favor of CombTG, compared to the CTG and BWSTTG $(p<0.016)$. However, no statistically significant difference was found in the mean change of CTG and BWSTTG ( $>0.05)$. There were statistically

Table 3. Mean change from baseline in balance and mobility parameters at the end of study in three groups

\begin{tabular}{|c|c|c|c|c|}
\hline & CombTG $(n=15)$ & & BWSTTG $(n=15)$ & \\
\hline & Mean \pm SD & Mean \pm SD & Mean \pm SD & $p$ \\
\hline Berg Balance Scale & $14.1 \pm 6.3$ & $8.7 \pm 2.4$ & $8.2 \pm 2.3$ & $\begin{array}{c}\mathbf{0 . 0 0 1}^{\star} \\
\text { CombTG-CGT } \mathbf{0 . 0 0 1} \\
\text { CombTG-BWSTTG } \mathbf{0 . 0 0 0 6} \\
\text { CGT-BWSTTG } 0.33\end{array}$ \\
\hline Single Leg Stance Test-affected side-a (sec) & $4.1 \pm 2.3$ & $1.6 \pm 1.7$ & $0.6 \pm 1.9$ & $\begin{array}{c}\mathbf{0 . 0 1 \dagger} \\
\text { CombTG-CGT } \mathbf{0 . 0 0 0 6} \\
\text { CombTG-BWSTTG }<\mathbf{0 . 0 0 1} \\
\text { CGT-BWSTTG } \mathbf{0 . 0 4 3}\end{array}$ \\
\hline Single Leg Stance Test-non-affected side-n (sec) & $13.7 \pm 10.1$ & $3.9 \pm 2.4$ & $2.8 \pm 3.1$ & $\begin{array}{c}\mathbf{0 . 0 4} \dagger \\
\text { CombTG-CGT } \mathbf{0 . 0 0 0 6} \\
\text { CombTG-BWSTTG }<\mathbf{0 . 0 0 1} \\
\text { CGT-BWSTTG } \mathbf{0 . 0 4 3}\end{array}$ \\
\hline Timed Get Up and Go Test (sec) & $-11.8 \pm 5.3$ & $-6.0 \pm 1.9$ & $-5.3 \pm 2.9$ & $\begin{array}{c}<\mathbf{0 . 0 0 1 \dagger} \\
\text { CombTG-CGT } \mathbf{0 . 0 0 7} \\
\text { CombTG-BWSTTG } \mathbf{0 . 0 0 7} \\
\text { CGT-BWSTTG } \mathbf{0 . 0 4 3}\end{array}$ \\
\hline Falls Efficiency Scale-International & $-13.2 \pm 7.1$ & $-5.1 \pm 3.4$ & $-5.3 \pm 1.9$ & $\begin{array}{c}<\mathbf{0 . 0 0 1}^{*} \\
\text { CombTG-CGT }<\mathbf{0 . 0 0 1}_{\text {CombTG-BWSTTG }<\mathbf{0 . 0 0 1}} \\
\text { CGT-BWSTTG } 0.33\end{array}$ \\
\hline Rivermead Mobility Index & $2.5 \pm 1.3$ & $1.5 \pm 0.9$ & $1.7 \pm 1.5$ & $\begin{array}{c}\mathbf{0 . 0 4 8}^{\star} \\
\text { CombTG-CGT } \mathbf{0 . 0 1 8} \\
\text { CombTG-BWSTTG } \mathbf{0 . 0 7 7} \\
\text { CGT-BWSTTG } 0.33\end{array}$ \\
\hline Comfortable 10-m Walk Test (sec) & $-5.9 \pm 4.7$ & $-2.9 \pm 1.4$ & $-3.1 \pm 2.1$ & $\begin{array}{c}\mathbf{0 . 1 3 5} \dagger \\
\text { CombTG-CGT } \mathbf{0 . 0 2} \\
\text { CombTG-BWSTTG } \mathbf{0 . 0 5} \\
\text { CGT-BWSTTG } 0.33\end{array}$ \\
\hline Stair Climbing Test (sec) & $-21.3 \pm 7.8$ & $-7.9 \pm 4.4$ & $-11.1 \pm 7.6$ & $\begin{array}{c}\mathbf{0 . 0 0 2} \dagger \\
\text { CombTG-CGT }<\mathbf{0 . 0 0 1} \\
\text { CombTG-BWSTTG }<\mathbf{0 . 0 0 1} \\
\text { CGT-BWSTTG } 0.33\end{array}$ \\
\hline
\end{tabular}

CombTG: Combined Training Group; CTG: Conventional Training Group; BWSTTG: Body Weight Supported Treadmill Training Group; SD: Standard deviation, sec: second;

* One-way ANOVA; $\dagger$ Kruskal-Wallis Test; Significant $p$ value adjusted for Bonferroni correction for pairwise comparisons ( $\mathrm{p}<0.016$ ). 
significant improvements $(\mathrm{p}<0.05)$ in the intergroup mean change of secondary outcome measures, except for CWT $(p=0.135)$. Moreover, the significance level of the RMI was tend to be statistically non-significant $(p=0.048)$. In the subgroup analysis, the FES-I and SCT outcome measures were found to be improved significantly in favor of the CombTG compared to the CTG and BWSTTG $(\mathrm{p}<0.016)$.

No adverse event was observed during the study period.

\section{DISCUSSION}

The results showed that CT, BWSTT, and CombTG increased balance and mobility and reduced fear of falling in post-stroke patients. Additionally, the CombTG experienced more improved balance and mobility performances than the CTG and BWSTTG. Despite different frequencies, both CT and BWSTT caused beneficial effects in the outcome measures.

It is not clear whether the improvements in balance in stroke patients are greater after the BWSTT, compared to other gait rehabilitation methods. ${ }^{[12]}$ Therefore, in this study, three groups with different training frequencies were included, and outcome measures were selected to evaluate the effects on both static and dynamic balance along with gait function. Also, as they were thought to be closely related to the balance function, the level of fear of falling, mobility, and stair climbing were assessed.

In previous studies which compared BWSTT with other gait training methods, therapy frequency of BWSTT was not found to be similar in each study. ${ }^{[11,29]}$ Dundar et al. ${ }^{[29]}$ concluded that balance function did not show a significant difference between the CTG (five times per week) and BWSTTG (three times CT per week plus two times BWSTT per week) after 30 sessions. In the present study, we found that CombTG (five times CT per week plus two times BWSTT per week during 30 sessions) improved more in balance than the CTG. Differently from their findings, our results showed that lower frequency of BWSTT induced similar effects with a higher frequency of CT. In another study which was designed as a crossover study on dependent ambulatory strokes, within the first four weeks, one of the group received only CT (five times per week), while the other group received both CT and BWSTT (three times per week). ${ }^{[1]}$ The authors concluded that there was no difference between the groups in the balance function after the trainings. The present study had similar groups (BWSTTG and CombTG); however, the CombTG improved more in balance performance than the BWSTTG. The main difference between two studies was the independence level of stroke patients' ambulation which may be considered as a factor to make a better use of BWSTT in terms of balance development.

There are some conflicting evidence about the isolated BWSTT in the literature. ${ }^{[1,29,30]}$ In one of these studies, isolated BWSTT and home-based training groups underwent 36 sessions of 90 -min training for 12 to 16 weeks. Similar improvements were reported in the walking speed, motor recovery, and balance in all groups. The authors concluded that the use of BWSTT was not superior to home-based progressive exercise training. ${ }^{[1]}$ In another study, it was shown that BWSTT might provide greater improvement on the TUG than home exercise training. ${ }^{[30]}$ In our study, both isolated BSWTT and CT had the same beneficial effects. As patients were supervised by a physical therapist during conventional program, this might have led to similar improvement with BSWTT. The high frequency of CT might be the second reason to provide the same effect with BSWTT.

There are some studies comparing the BWSTT with gait training programs other than CT. Bang and Shin ${ }^{[10]}$ compared the effects of BWSTT versus treadmill training on balance and gait parameters. The BWSTT was found more effective than treadmill training in improving balance and walking ability in patients with stroke. In another study, it was concluded that motor-learning science-based overground walking training was not superior to a BWSTT program of equal frequency and duration. ${ }^{[31]}$ Although the therapy frequency was the same in the groups, the results of the trials were not similar. In the present study, the therapy frequency was found to be superior in the CombTG compared to the CTG, and better balance function improvement was provided in the CombTG.

In the CombTG, the $10-\mathrm{m}$ comfortable walking time significantly decreased, compared to the other training groups. There are some studies which support our findings about walking speed. ${ }^{[10,16,30]}$ In contrast to our results, however, DePaul et al. ${ }^{[31]}$ showed that BWSTT had no superiority to overground walking training, when the trainings were delivered at the same frequency (15 sessions over five weeks). This conflict result has been interpreted by the authors as the effect of the more frequent training (30 sessions CT, 12 sessions BWSTT over six weeks) in the CombTG of our study.

After a stroke, falls are one of the most common medical complications with a $73 \%$ incidence within 
the first year. ${ }^{[32]}$ In addition to physical components, psychological factors related to balance impairment and falling include fear of falling and balance confidence. ${ }^{[33]}$ While fear of falling is defined as persisting concern regarding falling, balance confidence is defined as an individual's confidence in their ability to maintain their balance and remain steady. ${ }^{[34]}$ To the best of our knowledge, in the literature, there is no study investigating how the level of fear of falling changes after BWSTT in stroke patients; however, there are some studies concluding that balance confidence would be improved after BWSTT. ${ }^{[10,16]}$ In our study, the level of fear of falling significantly declined after training in all groups, although the CombTG had more significant change.

In the literature, no study is available researching the effects of how BWSTT would change the level of mobility and climbing stairs. The only study evaluating the performance standing on one leg showed that there was no significant difference after BWSTT compared to overground training. ${ }^{[13]}$ In this study, there were no data about on which leg the patients stood on. In our study, the performance was better in each group on both leg after training except that non-effected side test of BWSTTG. The CombTG's performance was significantly better, compared to other groups on both legs after training.

The study has important strengths in terms of the interpretation of the results. First, in the literature, the BWSTT efficacy trials were designed with only two study groups. However, our study design included three study groups. Second, this study provided scientific findings of different frequencies. Third, the patients were recruited from the same rehabilitation hospital in our study which provided homogeneity in both medical care and rehabilitation team. Finally, multiple outcome measures were used to assess all aspects of balance and fear of falling.

The clinical implication of these results indicated that lower frequency of isolated BWSTT was as much effective as higher frequency of $\mathrm{CT}$ on balance, mobility, and fear of falling in ambulatory post-stroke patients.

Nonetheless, there are some limitations to this study. First, the sample size is small. Second, there was no follow-up period in the study. Third, the study protocol included both inpatient and outpatient populations. Finally, there was a difference among the groups in terms of range of time after stroke, although there was no statistically significant difference among the groups in terms of the mean change.
In conclusion, this study demonstrates that CombTG has considerable effects on balance, mobility, and fear of falling parameters, while lower frequency of isolated BWSTT is as much effective as a higher frequency of CT in ambulatory post-stroke patients. Our results can contribute the effectiveness of isolated or combined training of BWSTT. The selection of appropriate treatment for balance improvement should be based on treatment equipment, available physiotherapist, and patient's motivation to participate in more frequent post-stroke rehabilitation. However, further comprehensive studies are required to evaluate the long-term effects of BWSTT on balance and mobility function of patients with stroke.

\section{Declaration of conflicting interests}

We thank to Saime Nilay Arman, Tugba Civi, and physiotherapist team of our hospital for their contributions to this study.

\section{Declaration of conflicting interests}

The authors declared no conflicts of interest with respect to the authorship and/or publication of this article.

\section{Funding}

The authors received no financial support for the research and/or authorship of this article.

\section{REFERENCES}

1. Duncan PW, Sullivan KJ, Behrman AL, Azen SP, Wu SS, Nadeau SE, et al. Body-weight-supported treadmill rehabilitation after stroke. N Engl J Med 2011;364:2026-36.

2. Pollock A, Baer G, Campbell P, Choo PL, Forster A, Morris J, et al. Physical rehabilitation approaches for the recovery of function and mobility following stroke. Cochrane Database Syst Rev 2014;4:CD001920.

3. Barbeau $H$. Locomotor training in neurorehabilitation: emerging rehabilitation concepts. Neurorehabil Neural Repair 2003;17:3-11.

4. Mao YR, Lo WL, Lin Q, Li L, Xiao X, Raghavan P, et al. The Effect of Body Weight Support Treadmill Training on Gait Recovery, Proximal Lower Limb Motor Pattern, and Balance in Patients with Subacute Stroke. Biomed Res Int 2015;2015:175719.

5. Mehrholz J, Thomas S, Elsner B. Treadmill training and body weight support for walking after stroke. Cochrane Database Syst Rev 2017;8:CD002840.

6. Lee HJ, Cho KH, Lee WH. The effects of body weight support treadmill training with power-assisted functional electrical stimulation on functional movement and gait in stroke patients. Am J Phys Med Rehabil 2013;92:1051-9.

7. Yen CL, Wang RY, Liao KK, Huang CC, Yang YR. Gait training induced change in corticomotor excitability in patients with chronic stroke. Neurorehabil Neural Repair 2008;22:22-30.

8. Mudge S, Rochester L, Recordon A. The effect of treadmill training on gait, balance and trunk control in a hemiplegic subject: a single system design. Disabil Rehabil 2003;25:1000-7. 
9. Trueblood PR. Partial body weight treadmill training in persons with chronic stroke. NeuroRehabilitation 2001;16:141-53.

10. Bang DH, Shin WS. Effects of robot-assisted gait training on spatiotemporal gait parameters and balance in patients with chronic stroke: A randomized controlled pilot trial. NeuroRehabilitation 2016;38:343-9.

11. Cho DY, Park SW, Lee MJ, Park DS, Kim EJ. Effects of robot-assisted gait training on the balance and gait of chronic stroke patients: focus on dependent ambulators. J Phys Ther Sci 2015;27:3053-7.

12. Swinnen E, Beckwée D, Meeusen R, Baeyens JP, Kerckhofs E. Does robot-assisted gait rehabilitation improve balance in stroke patients? A systematic review. Top Stroke Rehabil 2014;21:87-100.

13. Middleton A, Merlo-Rains A, Peters DM, Greene JV, Blanck EL, Moran R, et al. Body weight-supported treadmill training is no better than overground training for individuals with chronic stroke: a randomized controlled trial. Top Stroke Rehabil 2014;21:462-76.

14. Hsieh YW, Wu CY, Lin KC, Yao G, Wu KY, Chang YJ. Dose-response relationship of robot-assisted stroke motor rehabilitation: the impact of initial motor status. Stroke 2012;43:2729-34.

15. Lang CE, Lohse KR, Birkenmeier RL. Dose and timing in neurorehabilitation: prescribing motor therapy after stroke. Curr Opin Neurol 2015;28:549-55.

16. Combs SA, Dugan EL, Passmore M, Riesner C, Whipker D, Yingling E, et al. Balance, balance confidence, and healthrelated quality of life in persons with chronic stroke after body weight-supported treadmill training. Arch Phys Med Rehabil 2010;91:1914-9.

17. Vertesi A, Lever JA, Molloy DW, Sanderson B, Tuttle I, Pokoradi L, et al. Standardized Mini-Mental State Examination. Use and interpretation. Can Fam Physician 2001;47:2018-23.

18. Schwartz I, Sajin A, Fisher I, Neeb M, Shochina M, KatzLeurer $M$, et al. The effectiveness of locomotor therapy using robotic-assisted gait training in subacute stroke patients: a randomized controlled trial. PM R 2009;1:516-23.

19. Chang WH, Kim MS, Huh JP, Lee PK, Kim YH. Effects of robot-assisted gait training on cardiopulmonary fitness in subacute stroke patients: a randomized controlled study. Neurorehabil Neural Repair 2012;26:318-24.

20. Stevenson TJ. Detecting change in patients with stroke using the Berg Balance Scale. Aust J Physiother 2001;47:29-38.

21. Sahin F, Yilmaz F, Ozmaden A, Kotevolu N, Sahin T, Kuran
B. Reliability and validity of the Turkish version of the Berg Balance Scale. J Geriatr Phys Ther 2008;31:32-7.

22. Flansbjer UB, Blom J, Brogårdh $\mathrm{C}$. The reproducibility of Berg Balance Scale and the Single-leg Stance in chronic stroke and the relationship between the two tests. PM R 2012;4:165-70.

23. Flansbjer UB, Holmbäck AM, Downham D, Patten C, Lexell J. Reliability of gait performance tests in men and women with hemiparesis after stroke. J Rehabil Med 2005;37:75-82.

24. Podsiadlo D, Richardson S. The timed "Up \& Go": a test of basic functional mobility for frail elderly persons. J Am Geriatr Soc 1991;39:142-8.

25. Ulus Y, Durmus D, Akyol Y, Terzi Y, Bilgici A, Kuru O. Reliability and validity of the Turkish version of the Falls Efficacy Scale International (FES-I) in community-dwelling older persons. Arch Gerontol Geriatr 2012;54:429-33.

26. Akin B, Emiroglu ON 2007 The validity and reliability of the Turkish. version of Rivermead Mobility Index in the elderly. Turkish Journal of Geriatrics 2007;10:124-30.

27. Holden MK, Gill KM, Magliozzi MR, Nathan J, Piehl-Baker L. Clinical gait assessment in the neurologically impaired. Reliability and meaningfulness. Phys Ther 1984;64:35-40.

28. Kazis LE, Anderson JJ, Meenan RF. Effect sizes for interpreting changes in health status. Med Care 1989;27:178-89.

29. Dundar U, Toktas H, Solak O, Ulasli AM, Eroglu S. A comparative study of conventional physiotherapy versus robotic training combined with physiotherapy in patients with stroke. Top Stroke Rehabil 2014;21:453-61.

30. Uçar DE, Paker N, Buğdaycı D. Lokomat: a therapeuticchance for patients with chronic hemiplegia. NeuroRehabilitation 2014;34:447-53.

31. DePaul VG, Wishart LR, Richardson J, Thabane L, Ma J, Lee TD. Varied overground walking training versus bodyweight-supported treadmill training in adults within 1 year of stroke: a randomized controlled trial. Neurorehabil Neural Repair 2015;29:329-40.

32. Verheyden GS, Weerdesteyn V, Pickering RM, Kunkel D, Lennon S, Geurts AC, et al. Interventions for preventing falls in people after stroke. Cochrane Database Syst Rev 2013;5:CD008728.

33. Batchelor FA, Mackintosh SF, Said CM, Hill KD. Falls after stroke. Int J Stroke 2012;7:482-90.

34. Schinkel-Ivy A, Inness EL, Mansfield A. Relationships between fear of falling, balance confidence, and control of balance, gait, and reactive stepping in individuals with sub-acute stroke. Gait Posture 2016;43:154-9. 